\title{
Ebola Virus Requires Phosphatidylserine Scrambling Activity for Efficient Budding and Optimal Infectivity ${ }^{\dagger}$
}

\author{
Marissa Acciani ${ }^{1}$, Maria Lay ${ }^{1}$, Katherine E. Havranek ${ }^{1}$, Avery Duncan ${ }^{1}$, Hersha Iyer ${ }^{1}$, \\ Olivia Linn ${ }^{1}$ and Melinda Ann Brindley ${ }^{2, *}$ \\ 1 Department of Infectious Diseases, College of Veterinary Medicine, University of Georgia, \\ Athens, GA 30602, USA; marissa.acciani@uga.edu (M.A.); mfl71444@uga.edu (M.L.); \\ Katherine.havranek@uga.edu (K.E.H.); avery.duncan11@gmail.com (A.D.); hershaiyer@gmail.com (H.I.); \\ olivialinn8@gmail.com(O.L.) \\ 2 Department of Infectious Diseases, Department of Population Health, Center for Vaccines and \\ Immunology, College of Veterinary Medicine, University of Georgia, Athens, GA 30602, USA \\ * Correspondence: mbrindle@uga.edu \\ † Presented at Viruses 2020-Novel Concepts in Virology, Barcelona, Spain, 5-7 February 2020. \\ Published: 11 June 2020
}

\begin{abstract}
Ebola virus (EBOV) interacts with cells using multiple categories of cell-surface receptors, including C-type lectins and phosphatidylserine (PS) receptors. PS receptors typically bind to apoptotic cell membrane PS and orchestrate the uptake and clearance of apoptotic bodies. Many viruses coated with PS-containing lipid envelopes, acquired during budding from host cells, can also exploit these receptors for internalization. PS is restricted to the inner leaflet of the plasma membrane in homeostatic cells, an orientation that would be unfavorable for PS receptor-mediated uptake if conserved on the viral envelope. Therefore, it is theorized that viral infection induces hostcell PS externalization to the outer leaflet during replication. Cells have several membrane scramblase enzymes that enrich outer leaflet PS when activated. Here, we investigate the role of two scramblases, TMEM16F and XKR8, as possible mediators of cellular and viral envelope surface PS levels during recombinant vesicular stomatitis virus (VSV) in which the VSV glycoprotein was replaced with the Ebola glycoprotein (rVSV/EBOV-GP) replication and EBOV virus-like particle (VLP) production. We find that rVSV/EBOV-GP and EBOV VLPs produced in XKR8 knockout cells contain two- to threefold less PS in their outer leaflets. Consequently, rVSV/EBOV-GP produced in deltaXKR8 is $70 \%$ less efficient at infecting cells through apoptotic mimicry as compared to the viruses produced by parental cells. In addition, the budding efficiency of both recombinant VSV particles and VLPs was significantly reduced in cells lacking XKR8. Our data suggest that virion surface PS acquisition requires XKR8 activity, whereas the deletion of TMEM16F did not affect EBOV-GP-mediated entry of VLP production. Unexpectedly, we observed an additional role of XKR8 in rVSV/G, rVSV/EBOV-GP, and EBOV VLP budding.
\end{abstract}

Keywords: ebola virus; viral budding; lipid scramblase; XKR8; specific infectivity; phosphatidylserine 